\title{
Chemische und physiologische Studien über die Guanylsäure.
}

\author{
Von \\ Ivar Bang. \\ I. Theil. \\ Chemische Studien.
}

(Der Redaction zugegangen am 14. November 1900.)

Nach Kossèl1) können wir die Nucleinsäuren in drei Gruppen zusammenfassen : erstens Thymonucleinsäuren, zweitens Inosinsäure und Guanylsäure und drittens Plasminsäure.

Die Thymusnucleinsäuren sind nun unter sich verschiedenartig. Hier finden wir die beiden Nucleinsäuren der Thỵmusdrüse, die Nucleinsäuren aus dem Sperma der Fische und die Hefenucleinsäure. Die Gruppe wird am besten dadurch charakterisirt, dass der Kern dieser Nucleinsäuren aus einer (oder mehreren) substituirten Phosphorsäure in Verbindung mit der basischen Substanz Thymin als Thyminsäure besteht. Die Gruppe besteht also aus Nucleinsäuren, die sicher nahe verwandt sind, und ist als eine wohl charakterisirte zu bezeichnen. Man muss deshalb Kossel beistimmen, wenn er die Gruppe der Thymonucleinsäuren aufstellt.

Dagegen kann der Verfasser Kossel nicht weiter folgen, wenn er die Inosinsäure und Guanylsäure zu einer Gruppe zusammenfasst. Dies werde ich unten näher begründen. Auch ist es nach den vorliegenden Untersuchungen nicht sicher oder wahrscheinlich, dass die Plasminsäure eine echte Nucleinsäure ist; die dritte Gruppe Kossel's ist deshalb vielleicht auch nicht aufrecht $\mathrm{zu}$ halten.

Unter den Thymonucleinsäuren ist am längsten die Hefe-

1) Liebreich's Encyklopädie, Bd. 3, cit. noch Ascoli.

Hoppe-Seyler's Zeitschrift f. physiol. Chemie. XXXI. 
nucleinsäure bekannt. Diese Nucleinsäure ist zuerst von Kossel studirt worden; in einer kurzen Mittheilung ${ }^{1}$ ) hat er seine Erfahrungen über sie veröffentlicht, während eine ausführliche Publication niemals erschienen ist. Koss el findet die Zusammensetzung einer Formel $\mathrm{C}_{17} \mathrm{H}_{26} \mathrm{~N}_{6} \mathrm{P}_{2} \mathrm{O}_{14}$ od. $\mathrm{C}_{25} \mathrm{H}_{36} \mathrm{~N}_{9} \mathrm{P}_{3} \mathrm{O}_{20}$ entsprechend. Von den Spaltungsprodukten wurden Phosphorsäure, Xanthinbasen und eine reducirende Substanz erkannt, eine genauere Untersuchung dieser reducirenden Substanz ${ }^{2}$ ) lehrte, dass sie aus einer Pentose und einer Hexose bestand. Dies wurde durch fractionirte Krystallisation nachgewiesen. Leider hat Kossel uns weiter nichts über diese Pentose mitgetheilt, eine Schmelzpunktbestimmung des Osazons ausgenommen, und doch haben mehrere Forscher vergebens Zuckerarten und besonders Pentosen aus Nucleinsäuren und Nucloproteiden in Krystallen darzustellen versucht. Es ist deshalb schade, dass Kossel seine krystallisirte Pentose nicht näher beschrieben hat. Liebermann und v. Bitto ${ }^{3}$ ) haben später Einwände gegen die Existenz einer Pentose in der Hefenucleinsäure erhoben. In einer späteren Mittheilung ${ }^{4}$ ) hat auch Koss el seine Auffassung derart verändert, dass er jetzt die Zuckergruppe nicht mehr als einen Bestandtheil der Hefenucleinsäure ansieht, indessen dürfte doch nach den Untersuchungen von Herlant ${ }^{5}$ ) die Hefenucleinsäure dessenungeachtet eine Zuckergruppe enthalten.

Durch Alkalibehandlung konnte Kossel aus der Hefenucleinsäure mehrere neue Säuren darstellen, deren erste die Plasminsäure war, welche also damals ein Spaltungsprodukt war, jetzt aber als echte Nucleinsäure aufgestellt wird. Durch fortgesetzte Alkalibehandlung konnte man nach und nach alle organische Substanz abspalten und man bekam zuletzt eine anorganische Phosphorsäure, welche aber nicht eine gewöhnliche Metaphosphorsäure war, wie Kossel aus mehreren Reac-

1) Archiv f. Anatomie u. Physiologie. Phys. Abth. 1891.

2) Archiv f. Anatomie u. Physiologie. Phys. Abth. 1893.

3) Centralblatt f. Physiologie. 7. 1893.

4) Archiv f. Anatomie u. Physiologie. Phys. Abth. 1894.

5) Archiv f. exp. Path. u. Pharm. Bd. 44. 
tionen bewiesen hat. Merkwürdiger Weise hat neulich Ascoli ${ }^{1}$ ) in Kossel's Laboratorium gefunden, dass doch eine gewöhnliche Metaphosphorsäure hier vorliegt. Kossel's Beweise haben also einer Kritik nicht Stand halten können.

In Verbindung mit der Hefenucleinsäure dürfte auch die Plasminsäure besprochen werden. Diese Säure, die Kossel also als ein Spaltungsprodukt der Hefenucleinsäure beschrieben hat, ist jetzt von ihm in die Reihe der Nucleinsäuren gehoben. Wenn man aber bedenkt, dass diese Säure durch Salzsäurebehandlung dargestellt wird, ist die Sache gar nicht so einfach. Miescher hat ja gefunden, dass die Nucleinsäuren ausserordentlich schnell und leicht von Salzsäure gespalten werden, und es ist deshalb zu befürchten, dass die Salzsäurebehandlung hier schaden kann, sodass die Plasminsäure nicht als eine Nucleinsäure, sondern als Spaltungsprodukt anzusehen ist. Ihr kolossaler Phosphorgehalt spricht entschieden dafür.

Eine andere, von Kossel studirte Nucleinsäure ist die sogenannte Adenylsäure, oder wie sie jetzt heisst, die Thymusnucleinsäure.

Durch Behandlung der Thymusdrüse mit Baryt konnte Kossel eine Nucleinsäure darstellen, welche nur eine Xanthinbase enthielt, nämlich Adenin. Davon der Name Adenylsäure. ${ }^{2}$ ) Die Adenylsäure war sehr leicht zu zerlegen. Man bekam auch hier mehrere neue Säuren, zuerst die Paranucleinsäure, dann die Thyminsäure, welche in Thymin und Phosphorsäure zerlegt werden konnte.

Leider waren auch diese Untersuchungen nicht besser begründet wie die vorigen, da eine neue Untersuchung auch von $\mathrm{Koss} \mathrm{el}^{3}$ ) andere Resultate ergab. Die Adenylsäure enthielt nicht allein Adenin, sondern auch Guanin (und eine neue Base Cytosin), was Kossel merkwürdiger Weise bei seiner ersten Untersuchung nicht gefunden hatte. Ferner konnte Kossel seine Paranucleinsäure nicht wiederfinden. Die Para-

1) Zeitschrift f. phys. Chemie. Bd. XXVIII.

2) Archiv f. Anatomie u. Physiologie. Phys. Abth. 1894.

3) Zeitschrift f. phys. Chemie. Bd. XXII. 
nucleinsäure und Thyminsäure waren identische Substanzen. Als ein neuer Bestandtheil der Thymusnucleinsäure wurde ein Kohlehydrat gefunden, welches aber nicht als Zucker abgespalten wird und das als Lävulinsäure erkannt werden konnte. Die Kohlehydratgruppe war also sehr eng in der Nucleinsäure gebunden. Der Verfasser kann nur seine Verwunderung hierüber aussprechen, da die Thymusnucleinsäure nach Kossel so leicht in ihre übrigen Componenten Adenin, Guanin, Thymin, Phosphorsäure u. s. w. zerfällt. Wozu kann denn eigentlich die Kohlehydratgruppe gehören? (Weitere Untersuchungen besonders über die Thyminsäure sind hier erforderlich.)

Nach den letzten Untersuchungen der Thymusnucleinsäure von Neumann:) lässt sich aus den Thymusnucleinsäuren doch ein Zwischenstadium zwischen Nucleinsäure und Thyminsäure aufstellen, das Neumann Nucleothyminsäure nennt.

Als Typus der Nucleinsäuren von Fischen können wir die von Miescher und Schmiedeberg ${ }^{2}$ ) studirte Nucleinsäure aus Lachssperma oder die Salmonucleinsäure betrachten.

Die Zusammensetzung der Salmonucleinsäure entspricht einer Formel $\mathrm{C}_{40} \mathrm{H}_{56} \mathrm{~N}_{14} \mathrm{P}_{4} \mathrm{O}_{26}$. Die Säure besteht aus Thyminsäure oder Nucleotinphosphorsäure, wie Schmiedeberg sie nennt, welche mit den Xanthinbasen Adenin und Guanin verbunden ist. Die Verbindung ist eine salz-, nicht estherartige. Dies meint Schmiedeberg dadurch bewiesen zu haben, dass er aus Mischungen von Salmonucleinsäure und Xanthinbasen eine an Xanthinbasen reichere Substanz darstellen konnte, die sich ganz wie die Salmonucleinsäure verhält. Wenn man ferner zur Thyminsäure Xanthinbasen setzt, so bekommt man eine Säure, die sich wie die Salmonucleinsäure verhält. Sowohl die Thyminsäure als die Salmonucleinsäure verhindert den Nachweis zugesetzter Xanthinbasen durch Metallsalze. Kossel hat nun nachgewiesen, dass die Thymusnucleinsäure sich in dieser Beziehung ebenso wie die Salmonucleinsäure verhält,

1) Archiv f. Anatomie u. Physiologie. Phys. Abth. 1898-1899.

2) Archiv f. exp. Pathologie u. Pharm. Bd. 37 u. 43. 
er glaubt aber nicht, dass diese Nucleinsäure ihre Xanthinbasen in salzartiger Verbindung enthält. Dies beweist er ${ }^{1}$ ) auf folgende Weise: Setzte Kossel zu einer Lösung von Nucleinsäure Xanthinbasen, so konnte er durch Zusatz von Baryt und Alkohol die Nucleinsäure niederschlagen, während die Basen im Alkohol gelöst blieben. Ebenso verhielten sich Mischungen von Thyminsäure und Xanthinbasen, woraus Kos sel den Schluss zieht, dass "die Nucleinsäure keine derartig gebundenen Xanthinbasen enthält». Schmiedeberg hat es unterlassen, die Salmonucleinsäure und Thyminsäure ebenso zu untersuchen, und wir können deshalb nicht wissen, ob die Theorie der salzartigen Bindung der Xanthinbasen in der Salmonucleinsäure richtig ist. Da nun aber Schmiedeberg's Schüler Herlant ${ }^{1}$ ) die Identität der Salmonucleinsäure und Thymusnucleinsäure sehr wahrscheinlich gemacht hat, so stehen die Auffassungen Kossel's und Schmiedeberg's in Widerspruch.

Kann man nun nicht die obige Auffassung Schmiedeberg's ohne Weiteres acceptiren, so steht auch die Frage nach der Zusammensetzung der Salmonucleinsäure bis auf Weiteres offen. Wir kennen eben so wenig die Constitution dieser Nucleinsäure als der übrigen oben erwähnten.

Die Constitution der nächsten Nucleinsäure, der Inosinsäure, ist dagegen ziemlich klar. Diese Säure, von Liebig und Haiser ${ }^{2}$ ) gefunden und untersucht, hat eine einfache Formel $\mathrm{C}_{10} \mathrm{H}_{13} \mathrm{~N}_{4} \mathrm{PO}_{8}$ und ist aus einer Phosphorsäure, welche mit Hypoxanthin und wahrscheinlich 0xyvaleriansäure verbunden ist, zusammengesetzt. Die Inosinsäure hat also eine sehr einfache Constitution.

Die letzte Nucleinsäure ist die Guanylsäure, welche vom Verfasser ${ }^{3}$ ) untersucht und beschrieben worden ist. Da diese Nucleinsäure in der vorliegenden Abhandlung der Gegenstand unserer Untersuchung sein soll, dürfte es zweck-

1) 1. c.

2) Monatsh. f. Chemie Bd. 16.

3) Zeitschr. f. physiol. Chemie Bd. XXV. 
mässig sein, erst die früher erhaltenen Resultate zu recapituliren.

Die Guanylsäure lässt sich aus dem Pancreasproteid darstellen, man kann aber auch das Pancreas selbst hierzu benutzen. Da sie sehr fest mit dem Eiweiss verbunden ist, muss man das Proteid mit Alkalilauge kochen, um die Verbindung zu spalten. Man kann dies allerdings auch durch Kochen mit Wasser allein erzielen, die Ausbeute wird aber dann sehr schlecht.

Die Guanylsäure ist sehr leicht in heissem Wasser löslich, dagegen ist die Löslichkeit in kaltem Wasser verhältnissmässig klein. Da nun die Guanylsäure gegen die Einwirkung heissen $\mathrm{W}^{\top}$ assers resistent ist, so kann man sie zweckmässig durch wiederholtes Lösen in heissem Wasser von den Verunreinigungen befreien. Die Guanylsäure ist in Mineralsäuren und Alkalien löslich, dagegen ist sie in verdünnter Essigsäure schwer löslich. Auch wird sie von Essigsäure aus ihrer Lösung in Alkali niedergeschlagen.

Die Guanylsäure verbindet sich mit Metalloxyden zu Salzen, die gewöhnlich unlöslich oder schwerlöslich im Wasser sind.

Die Zusammensetzung der Säure war im Durchschnitt $34,18 \% \mathrm{C}, 4,430 \mathrm{H}, 18,21 \% \mathrm{~N}$ und $7,64 \% \mathrm{P}$. Diese Zusammensetzung stimmte am besten mit einer Formel $\mathrm{C}_{22} \mathrm{H}_{34} \mathrm{~N}_{10} \mathrm{P}_{2} \mathrm{O}_{17}$ überein.

Von den Spaltungsprodukten wurde eine Pentose erkannt, welche in einer Menge von ca. 30\% vorkommt, als Traubenzucker berechnet. Weiter enthielt die Guanylsäure mindestens 35\% Guanin. Ausser Guanin wurde keine andere Xanthinbase gefunden. Als drittes Spaltungsprodukt wurde Ammoniak gefunden. Doch konnte der Verfasser nicht mit Bestimmtheit die Möglichkeit abweisen, dass das Ammoniak ein secundäres Spaltungsprodukt war. Im Gegentheil war dies nicht unwahrscheinlich. Das Thymin wurde unter den Spaltungsprodukten vermisst. Im Ganzen wurde also von den Spaltungsprodukten etwa $85 \%$ wiedergefunden, die Phosphorsäure mitgerechnet. Es blieb also ein Deficit von etwa 15\%, 
das aus unbekannten Substanzen bestand, abgesehen davon, dass die Spaltung wahrscheinlich eine hydrolytische ist. Da ich damals versprochen hatte, diese Spaltungsprodukte zu erforschen, so will ich jetzt mein Versprechen erfüllen.

Die Aufgaben, welche noch zur Erforschung der Guanylsäure vorliegen, sind die folgenden :

1. Durch die Analyse einiger Salze der Säure die empirische Formel festzulegen.

2. Man muss entscheiden, $o b$ das Ammoniak ein primäres oder secundäres Spaltungsprodukt ist.

3. Es müssen die unbekannten. Spaltungsprodukte erforscht werden, und wenn sämmtliche Componenten der Guanylsäure gefunden sind, kann man zur Aufstellung der rationellen Formel übergehen.

Einige Fragen stehen noch aus, z. B. über die Natur der Pentose, wir werden aber diese nicht berühren. Nachdem ich mir eine neue Portion Guanylsäure dargestellt hatte, wurde erst die Zusammensetzung untersucht. Eine N-Analyse ergab 18,08\% N, eine C- und H-Bestimmung 34,18\% $\mathrm{C}$ und 4,53\% H. Die Analysen stimmen somit mit den vorigen, und wir können das Guanylsäurepräparat als rein bezeichnen. Von diesem Präparate wurde das Silbersalz dargestellt durch Fällung des Guanylsäurealkali mit Silbernitrat. Der Niederschlag, welcher lichtempfindlich ist, wurde ausgewaschen, getrocknet und pulverisirt. Da das Silbersalz phosphorhaltig ist, so kann man das Silber nicht allein durch Glühen bestimmen. Ich veraschte deshalb das Präparat, zog es mit kochender Salpetersäure so oft aus, als das Filtrat noch eine Silberreaction gab, und schlug das Silber mit Chlornatrium nieder. Das Chlorsilber wurde auf gewöhnliche Weise bestimmt.

\footnotetext{
1. 0,17כ̃2 g Substanz abgewogen $0,0594 \mathrm{~g} \mathrm{AgCl}$$$
0,0441 \mathrm{~g} \mathrm{Ag}=\mathbf{2 5}, \mathbf{5} 2 \% \text {. }
$$

2. $0,2260 \mathrm{~g}$ Substanz abgewogen $0,0772 \mathrm{~g} \mathrm{AgCl}$$$
0,0581 \mathrm{~g} \mathrm{Ag}=25,71 \% \text {. }
$$

3. 0,2385 g Substanz abgewogen

$0,0811 \mathrm{~g} \mathrm{AgCl}$

$0,0612 \mathrm{~g} \mathrm{Ag}=25,60 \%$.
} 
Im Durchschnitt enthält das Silbersalz 25,61\% Ag. Die Molekularzahl des Silbersalzes wird demgemäss 431 und die der Guanylsäure 314.

Es lässt sich aber leicht zeigen, dass die Molekularzahl der Guanylsäure grösser sein muss als 314 , da wir ja $7,64 \% \mathrm{P}$ gefunden haben, was einer Zahl der P-Atome von 0,78 entspricht, wenn wir eine Molekularzahl von 314 voraussetzen. Folglich muss die Guanylsäure eine mehrbasische Säure sein. Die Molekularzahl ist deshalb ein Multiplum von 314, oder $n \times 314$.

Setzen wir $\mathrm{n}=2$, wird die Zahl der Atome des Phosphors 1,$56 ; \mathrm{n}=3$ gibt $\mathrm{P}=2,34, \mathrm{n}=4$ fordert $\mathrm{P}=\mathbf{3 , 1 2}$ und $\mathrm{n}=5, \mathrm{P}=\mathbf{3 , 9 0}$.

Man kann hieraus den Schluss ziehen, dass $n$ wahrscheinlich 4 oder 5 ist, da nur diese Zahlen einen passenden Werth für den Phosphor geben. Die Molekularzahl wird deswegen entweder 1236 oder 1570.

Dass die erste Molekularzahl 1236 unmöglich oder jedenfalls wenig wahrscheinlich ist, geht aus der entsprechenden Formel hervor. Diese Formel wird nämlich

$$
\mathrm{C}_{35},{ }_{79} \mathrm{H}_{57,40} \mathrm{~N}_{16,34} \mathrm{P}_{3,10} \mathrm{O}_{27,79} \text {. }
$$

Setzen wir dagegen die Molekularzahl zu 1570, so bekommen wir die Formel $\mathrm{C}_{44,72} \mathrm{H}_{71,75} \mathrm{~N}_{20,42} \mathrm{P}_{3,{ }_{37}} \mathrm{O}_{35}, 26$, also auch nicht eine besonders gute Uebereinstimmung.

Nehmen wir aber an, dass wir etwa $0,3 \%$ zu wenig Silber wiedergefunden haben, was nicht unmöglich ist, wenn man das ungewöhnlich complicirte Verfahren zur Bestimmung des Silbers bedenkt, so werden die Molekularzahlen entweder 1236 oder 1545. Die Molekularzahl 1236 gibt dann eine Formel $\mathrm{C}_{35,20} \mathrm{H}_{56,49} \mathrm{~N}_{16,08} \mathrm{P}_{3,05} \mathrm{O}_{27,35}$, welche mit einer Formel $\mathrm{C}_{35} \mathrm{H}_{56} \mathrm{~N}_{16} \mathrm{P}_{3} \mathrm{O}_{27}$ übereinstimmt. Die Molekularzahl 1545 dagegen gibt eine Formel $\mathrm{C}_{44,01} \mathrm{H}_{70,60} \mathrm{~N}_{20,09} \mathrm{P}_{3,81} \mathrm{O}_{34},{ }_{19}$ also abgerundet: $\mathrm{C}_{44} \mathrm{H}_{70} \mathrm{~N}_{20} \mathrm{P}_{4} \mathrm{O}_{34}$.

Wir werden jetzt untersuchen, wie diese Formel mit der procentischen Zusammensetzung harmonirt. 


\begin{tabular}{|c|c|c|}
\hline $\begin{array}{l}\text { Berechnet für } \\
\mathrm{C}_{35} \mathrm{H}_{56} \mathrm{~N}_{16} \mathrm{P}_{3} \mathrm{O}_{87}\end{array}$ & $\begin{array}{l}\text { Berechnet für } \\
\mathrm{C}_{44} \mathrm{H}_{70} \mathrm{~N}_{80} \mathrm{P}_{4} \mathrm{O}_{84}\end{array}$ & Gefunden \\
\hline C $\quad 34,29 \%$ & $34,15 \%$ & $34,18 \%$ \\
\hline $4,57 \%$ & $4,53 \%$ & $4,57 \%$ \\
\hline $18,29 \%$ & $18,11 \%$ & $18,21 \%$ \\
\hline $7,59 \%$ & $8,02 \%$ & $7,64 \%$ \\
\hline
\end{tabular}

Man sieht also, dass beide Formeln ganz gut mit der procentischen Zusammensetzung harmoniren, die erste Formel vielleicht am besten. Indessen wird der Verfasser sich entschieden für die zweite Formel aussprechen. Es ist nämlich wenig wahrscheinlich, dass man zuviel Phosphor gefunden hat, dagegen hat ein theoretischer Phosphorwerth von $8,02 \%$ viel mehr für sich und stimmt ungefähr mit dem, was man durch die P-Analysen erwarten kann. Der Verfasser glaubt deswegen, dass die Formel $\mathrm{C}_{44} \mathrm{H}_{70} \mathrm{~N}_{20} \mathrm{P}_{4} \mathrm{O}_{34}$ am besten stimmt sowohl mit den Elementar-Analysen als auch mit der Berechnung des Silbersalzes. Es lässt sich auch leicht zeigen, dass diese Formel keine Variation der Zahlen der Atome des C, N, P und.zulässt. Nur einige Variationen des Wasserstoffes sind möglich. Die Formel $\mathrm{C}_{44} \mathrm{H}_{70} \mathrm{~N}_{20} \mathrm{P}_{4} \mathrm{O}_{34}$ ist genau zweimal so gross, als die erste vom Verfasser aufgestellte Formel $\mathrm{C}_{22} \mathrm{H}_{34} \mathrm{~N}_{10} \mathrm{P}_{2} \mathrm{O}_{17}$.

Wir werden unten zeigen, dass die H-Atome nicht 70, aber wahrscheinlich 66 sind. Die endliche Formel der Guanylsäure ist deshalb:

$$
\mathrm{C}_{44} \mathrm{H}_{66} \mathrm{~N}_{20} \mathrm{P}_{4} \mathrm{O}_{34} \text {. }
$$

Weiter kann man hieraus folgern, dass die Guanylsäure eine 5-basische Nucleinsäure ist. Das Silbersalz hat also die folgende Zusammensetzung:

$$
\mathrm{C}_{44} \mathrm{H}_{61} \mathrm{Ag}_{5} \mathrm{~N}_{20} \mathrm{P}_{4} \mathrm{O}_{34}
$$

Wenn die Formel zeigt, dass die Guanylsäure 20 Atome Stickstoff enthält, so wird die Existenz des Ammoniaks als primäres Spaltungsprodukt der Guanylsäure sehr zweifelhaft. Wäre dies der Fall, so muss man eine Formel mit mindestens 90 Atomen $\mathrm{N}$ annehmen. Wir werden diese Frage genauer untersuchen.

Meine erste publicirte Untersuchung über die Spaltungsprodukte der Guanylsäure haben einen vorläufigen Ammoniak- 
werth von etwa $0,8 \%$ des Stickstoffes. Indessen war diese Zahl sehr unsicher, da nur ca. 0,1 g Guanylsäure zu der Bestimmung von $\mathrm{NH}_{3}$ nach Schlösing benutzt worden war.

Ich habe diesmal erstens eine grössere Guanylsäuremenge benützt und zweitens habe ich die Methode verbessert.

Nachdem ich nämlich die Guanylsäure mit 5\% iger Schwefelsäure 3 Stunden gekocht hatte, wurde die Lösung in einen Destillationskolben übergeführt und mit einem Ueberschuss von Magnesia destillirt. Selbstverständlich habe ich durch Versuche mit Guaninsulfat (aus dem Pancreasproteid dargestellt) die Methode ausprobirt und anwendbar gefunden.

0,3984 g Guanylsäure (Präp. Nr. IV) wurde mit $100 \mathrm{ccm}$. $5 \%$ iger Schwefelsäure 3 Stunden auf dem Wasserbade gekocht und danach mit $10 \mathrm{~g} \mathrm{MgO}$ destillirt. $\mathbf{0 , 3 3} \mathrm{ccm}$. Titrirschwefelsäure wurde verbraucht, was $0,00046 \mathrm{~g} \mathrm{~N}=$ etwa $0,11 \% \mathrm{~N}$ entspricht.

$0,6684 \mathrm{~g}$ Guaninsulfat wurde 3 Stunden mit $100 \mathrm{ccm}$. $5 \%$ iger Schwefelsäure gekocht und danach mit $10 \mathrm{~g} \mathrm{MgO}$ destillirt. Die gefundene $\mathrm{N}$-Menge war 0,0008 g.

Man kann aus diesen Versuchen den Schluss ziehen, dass eine sehr kleine Ammoniakmenge aus der Guanylsäure durch Kochen mit 5\% iger Schwefelsäure entsteht. Diese $\mathrm{NH}_{3}$-Menge entspricht aber ungefähr dem, was aus Guanin durch Kochen mit 5\% iger Schwefelsäure entsteht. Das Ammoniak ist deshalb kein primäres Spaltungsprodukt, entsteht dagegen wahrscheinlich aus dem gebildeten Guanin.

Wenn dies der Fall ist, kann man mit grosser Wahrscheinlichkeit auch andere stickstoffhaltige Spaltungsprodukte ausschliessen, absolut sicher ist man aber nicht.

Wenn man davon ausgeht, dass Guanin das einzige N-haltige Spaltungsprodukt ist, enthält die Guanylsäure 39,29\% Guanin, während wir nur etwa $35 \%$ gefunden haben. ${ }^{1}$ ) Indessen wissen wir, dass ein wenig Guanin durch das Kochen mit Mineralsäure zerstört worden ist. Auch ist die Fällung

1) Bang, l. c. 
mit ammoniakalischem Silber nicht ganz absolut. Etwas Guanin ist deshalb wahrscheinlich verloren gegangen. Es lässt sich aber einigermassen berechnen, wieviel Guanin man nach dem Kochen mit der Mineralsäure durch die ammoniakalische Silberfällung wiederfinden kann. Ich habe zwei solche Versuche ausgeführt. Abgewogene Mengen Guanin wurden 3 Stunden mit 5\% iger Schwefelsäure gekocht und danach mit Silbernitrat und Ammoniak gefällt. Der Niederschlag wurde getrocknet, gewogen und auf Guanin umgerechnet.

1. $0,1222 \mathrm{~g}$ Guanin abgewogen. $0,1208 \mathrm{~g}$ wiedergefunden $=98,16^{\circ} \%$

2. $0,114 \mathrm{~g}>>0,1114 \mathrm{~g} \quad>\quad=97,38 \%$

Im Durchschnitt wiedergefunden $=97,77 \%$

Nimmt man an, dass man auch bei den Guanylsäureversuchen etwa 97,77\% wieder gefunden hat, wird das Resultat der Guaninbestimmungen 36,55\% Guanin, also 2,74\% weniger als der theoretische Werth, und doch haben wir nur zwei Analysen ausgeführt und mit sehr kleinen Substanzmengen. Leider habe ich diesmal nicht mehrere Guaninbestimmungen ausführen können. Es ist aber nach meiner Ansicht ziemlich gut festgestellt, dass aller Stickstoff sich als Guanin in dem Guanylsäuremolekül vorfindet. Die Guanylsäure enthält demgemäss 4 Moleküle Guanin.

Es lässt sich hieraus berechnen, wie viel Zucker die Guanylsäure enthalten muss.

$$
\begin{aligned}
& \text { 39,3\% Guanin : } 30 \% \text { Zucker }=4(1.51): X(150) \\
& X=3,07 \text {. }
\end{aligned}
$$

Folglich enthält die Guanylsäure 3 Moleküle Zucker als Pentose berechnet. Nun haben wir aber die Pentose immer als Traubenzucker berechnet, da die hypothetische Pentose in Beziehung auf Reductionsvermögen uns nicht bekannt ist. Nehmen wir an, dass unsere Pentose dasselbe Reductionsvermögen wie die Xylose hat, so haben wir nur 27\% Pentose titrimetrisch bestimmt gefunden. Dies entspricht einer Zahl der Moleküle der Zuckergruppe von 2,76. Nun habe ich aber nachgewiesen, dass man niemals durch Kochen der Guanylsäure mit einer Mineralsäure die ganze Zuckermenge finden kann, da die Bildung von Zucker immer von einer fortlaufen- 
den Zerstörung des Zuckers begleitet ist. ${ }^{1}$ ) Wenn man deshalb einen theoretischen Zuckerwerth von 29\% anstatt 27\% supponirt, wird die Molekularzahl 2,97, also ganz genau 3 Moleküle Zucker.

Bei dieser Berechnung haben wir immer vorausgesetzt, dass unsere Zuckerart eine Pentose sei. Und in der That ist es viel wahrscheinlicher, dass wir eine Pentose mit grösserem Reductionsvermögen vor uns haben, da wir, wie oben erwähnt, sicher zu wenig Zucker bei unseren Analysen gefunden haben und demgemäss aus diesen Analysen einen kleinen Multiplicator finden müssen als umgekehrt.

Gehen wir nun zu den bis jetzt unbekannten Spaltungsprodukten über, so werden wir die völlige Bestätigung unserer obigen Deductionen finden.

War die Formel der Guanylsäure $\mathrm{C}_{44} \mathrm{H}_{66} \mathrm{~N}_{20} \mathrm{P}_{4} \mathrm{O}_{34}$, so haben wir unter den Spaltungsprodukten, abgesehen von dem Phosphor, gefunden: $4 \mathrm{C}_{5} \mathrm{H}_{5} \mathrm{~N}_{5} \mathrm{O}+3 \mathrm{C}_{5} \mathrm{H}_{10} \mathrm{O}_{5}$, zusammen $35 \mathrm{C}$ und $20 \mathrm{~N}$. Als Rest bleiben also $9 \mathrm{G}$ und kein $\mathrm{N}$. Die letzten Spaltungsprodukte enthalten folglich 9 Atome C, sind aber stickst offfrei.

Es hat sich herausgestellt, dass das letzte SpaItungsprodukt der Guanylsäure Glycerin ist.

Die Existenz des Glycerins in der Guanylsäure wurde auf folgende Weise constatirt:

Nachdem ich die oben erwähnte Guanylsäure $(0,3984 \mathrm{~g})$ mit Magnesia destillirt hatte, wurde der Rest mehrmals mit 97\%igem Alkohol ausgezogen. Der Alkohol wurde langsam verdunstet. Das Residuum wurde nach König ${ }^{2}$ ) auf Glycerin untersucht.

Es wurde mit Quarzsand und Kalkmilch eingedampft und dann mit heissem absoluten Alkohol extrahirt. Das alkoholische Filtrat wurde nach 24 Stunden filtrirt und das neue Filtrat vorsichtig eingedampft und aufs Neue mit absolutem Alkohol extrahirt. Der Alkohol wurde mit Aether versetzt

1) Bang, 1. c.

2) Untersuchung landwirthsch. $u$. gewerbl. wichtiger Stoffe. 
und nach einigen Stunden filtrirt. Der Aetheralkohol wurde verjagt und es blieb ein Syrup zurück.

Dieser Syrup hatte folgende Eigenschaften:

Er schmeckte schwach süss, reducirte aber nicht Kupferoxyd und Alkali.

Er gab die Acrolein reaction durch Erhitzen mit Kaliumbisulfat. Eine Verkohlung fand hierbei nicht statt. Die Lösung ist also zuckerfrei.

Er gab eine grüne Boraxperle.

Man kann hieraus schliessen, dass Glycerin vorlag. Die Existenz des Glycerins wurde dennoch weiter gesichert. Die Lösung wurde mit Alkali und überschüssigem Permanganat versetzt und eine halbe Stunde gekocht. Die blaue Lösung wurde dann mit $\mathrm{SO}_{2}$ entfärbt und filtrirt. Das Filtrat versetzte ich mit $\mathrm{CaCl}_{2}$ und bekam eine krystallinische Fällung. . Diese Fällung wurde mit Schwefelsäure versetzt und schwach erhitzt. Eine zugesetzte verdünnte Permanganatlösung wurde sofort entfärbt. Folglich enthielt die Lösung 0xalsäure, nach FoxWanklyn und Benedikt-Zsigmondy ${ }^{1}$ ) ein charakteristisches Spaltungsprodukt des Glycerins durch Oxydation mit Permanganat.

Die Guanylsäure enthält also Glycerin und die Existenz des Glycerins wurde weiter durch die Acroleinreaction in mehreren Guanylsäurepräparaten gesichert. Zwar ist der Zucker der Guanylsäure bei der Acroleinreaction lästig, man kann aber sehr deutlich nach der Zerstörung des Zuckers durch Kaliumbisulfat das Acrolein erkennen.

Man kann nun den Einwand machen, dass die Guanylsäure vielleicht durch Fett oder Lecithin verunreinigt war. Hierzu ist zu bemerken, dass ich die Guanylsäurepräparate mit Alkohol und Aether extrahirte, bis diese Lösungsmittel nichts mehr aufnahmen. Der Einwand ist folglich hinfällig.

Nachdem wir die Existenz des Glycerins erkannt haben, bleibt noch zu untersuchen, ob das Glycerin das letzte Spaltungsprodukt der Guanylsäure ist, oder ob diese Nucleinsäure

1) Zeitschr. f. analyt. Chemie. Bd. 25. 
vielleicht noch mehrere solche enthält. Ich habe zum Vergleich der Glycerinmenge einen Parallelversuch mit 0,05 g Glycerin in $100 \mathrm{ccm}$. Wasser ausgeführt. Diese Lösung wurde ganz wie die der Guanylsäure behandelt. Durch Titrirung der gebildeten Oxalsäure konnte man ungefähr bestimmen, dass die Lösung der Guanylsäure nicht weniger Glycerin als 0,05 g Glycerin enthielt. Die Guanylsäure enthält demgemäss mindestens ca. $12 \%$ Glycerin.

Man kann daraus sicher den Schluss ziehen, dass das Glycerin das letzte Spaltungsprodukt der Guanylsäure ist.

Die Zusammensetzung der Guanylsäure ist hiermit erforscht, und wir können jetzt die Constitution feststellen.

Die Guanylsäure enthält: 4 Moleküle Guanin, 3 Moleküle Zucker, 3 Moleküle Glycerin und 4 Moleküle Phosphorsäure. Nehmen wir an, dass die Spaltung der Guanylsäure durch Kochen mit einer Mineralsäure eine hydrolytische ist, so muss sie auf folgende Weise verlaufen:

$$
\begin{aligned}
\mathrm{C}_{44} \mathrm{H}_{66} \mathrm{~N}_{20} \mathrm{P}_{4} \mathrm{O}_{34}+10 \mathrm{H}_{2} \mathrm{O} & =4 \mathrm{C}_{5} \mathrm{H}_{5} \mathrm{~N}_{5} \mathrm{O}+3 \mathrm{C}_{5} \mathrm{H}_{10} \mathrm{O}_{5}+3 \mathrm{C}_{3} \mathrm{H}_{8} \mathrm{O}_{3} \\
& \left.+4 \mathrm{H}_{3} \mathrm{PO}_{4} \cdot{ }^{1}\right)
\end{aligned}
$$

Da wir nun 4 Moleküle Phosphorsäure und 4 Moleküle Guanin haben, so können wir annehmen, dass wir 4 mit einander verbundene Phosphorsäuren besitzen, jede mit einem Molekül Guanin verbunden. Die 3 Moleküle Glycerin sind wahrscheinlich sowohl mit den Phosphorsäuren als Glycerinphosphorsäure als auch mit den 3 Molekülen Zucker verkettet. 3 Moleküle Phosphorsäure sind also sowohl mit Guanin als Glycerin verbunden. 1 Molekül Phosphorsäure ist nur mit Guanin verbunden. Endlich erinnern wir uns, dass die Guanylsäure eine 5-basische Säure ist.

Auf diese Voraussetzungen bauend, denke ich mir die Constitution der Guanylsäure folgendermassen:

1) Selbstverständlich kann hier eine Anhydridform der Phosphorsäure ebenso gut vorkommen. Nur braucht man dann weniger $\mathrm{H}_{2} \mathrm{O}$ zur Hydrolyse. 


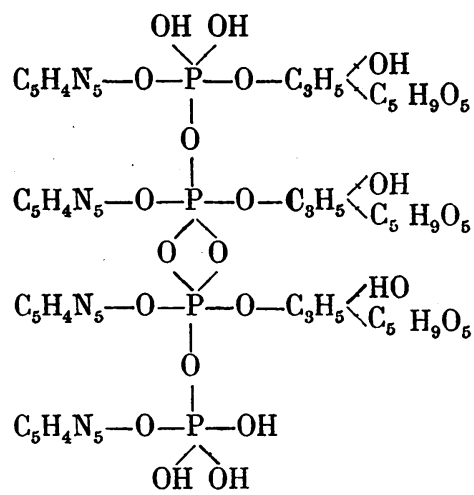

Die Erkenntniss der Constitution der Guanylsäure bietet in mehreren Beziehungen Interesse dar. Abgesehen von der Thatsache, dass hierdurch zum ersten Mal eine Nucleinsäure vollständig untersucht und beschrieben worden ist (die Inosinsäure ist nicht so vollständig untersucht), haben wir bewiesen: 1. Die Guanylsäure enthält nur eine Xanthinbase. Aller Stickstoff wird von dem Guanin geliefert. 2. Die Guanylsäure enthält eine Pentosegruppe. Salkowski's Untersuchung des Osazons hat hierdurch eine Bestätigung bekommen. Niemand kann jetzt an der Existenz der Pentose im Pancreasproteid und in der Guanylsäure zweifeln. 3. Die Guanylsäure enthält Glycerin, das als Glycerinphosphorsäure vorkommt. Die Guanylsäure bekommt hierdurch ein besonderes Interesse. Sie stimmt nämlich mit dem Lecithin sehr überein. Beide haben als Kern Glycerinphosphorsäure, welche im Lecithin mit Fettsäure und Cholin verbunden ist, in der Guanylsäure aber mit Zucker und Guanin. Es ist auch nicht unwahrscheinlich, dass diese Substanzen auch mit einander verwandt sind. Licithin wird ja allgemein als Baumaterial der Nucleoproteide bezw. Nucleinsäuren angesehen. Während sonst die Lecithine bis zum Phosphor gespalten werden, bleibt hier die ganze Glycerinphosphorsäure zum Aufbau der Guanylsäure angewandt. Es ist selbstverständlich, dass man nach dem Vorkommen von Glycerin in der Guanylsäure auch anderswo besonders unter den Nucleinsäuren und Nucleoproteiden nach Glycerin suchen muss. Man kann ja möglicher 
Weise auch Substanzen begegnen, welche noch näher mit Lecithin übereinstimmen. Die Lecithalbumine, Ovovitelline etc. können vielleicht Glieder derselben Gruppe wie die Nucleoproteide sein. Als Zwischenglieder haben wir dann Substanzen, welche dem Lecithalbumine näher oder ferner stehen. Jedenfalls dürfte die Zusammensetzung der Guanylsäure $\mathrm{zu}$ einer wiederholten Untersuchung der Lecithalbumine und verwandter Substanzen anregen.

In Vergleich mit den übrigen Nucleinsäuren nimmt die Guanylsäure eine Sonderstellung ein. Es ist nicht mit Kossel richtig, dieselbe in einer gemeinsamen Gruppe mit der Inosinsäure zusammenstellen, da diese Nucleinsäuren nicht mit einander näher verwandt sind. Keine anderen Nucleinsäuren haben solche physikalischen und chemischen Eigenschaften, keine haben eine ähnliche Zusammensetzung. Auch ist die empirische Formel ziemlich verschieden.

Stellen wir die Nucleinsäuren nach ihrer Formel zusammen, so bekommen wir folgende Tabelle:

$\begin{array}{lccrcr} & \text { C } & \text { H } & \text { N } & \text { P } & \text { 0 } \\ \text { Inosinsäure . . . . } & 10 & 13 & 4 & 1 & 8 \\ \text { Salmonucleinsäure } & 40 & 56 & 14 & 4 & 26 \\ \text { Hefenucleinsäure ') } & 40 & 60 & 16 & 4 & 32 \\ \text { Guanylsäure . . } & 44 & 66 & 20 & 4 & 34\end{array}$

(Die ersten von Kossel veröffentlichten Formeln über Hefenucleinsäure und Thymusnucleinsäure sind wahrscheinlich unrichtig; sie werden deshalb nicht mitgenommen).

Man sieht, dass die Zahlen ziemlich weit von einander differiren. Constant ist allein der Phosphor (abgesehen von der Inosinsäure). Alle drei Nucleinsäuren bestehen aus 4 Molekülen Phosphorsäure. Dagegen ist das Verhältniss $\mathrm{N}: \mathrm{P}$ sehr wechselnd, ein Verhältniss von $3 \mathrm{~N}: 1 \mathrm{P}$, wie Kossel es als das Gewöhnliche annimmt, kommt überhaupt nicht vor. Eine Uebersichtstabelle wird dies erleuchten:

1) Analyse von Miescher. Nach Schmiedeberg waren aber die Präparate nicht ganz rein. 


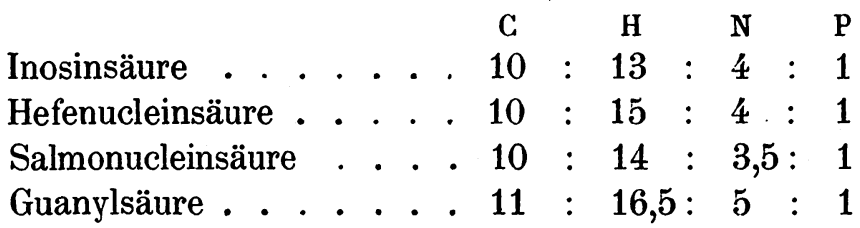

Man sieht, wie sehr das Verhältniss der Zahlen variirt. Am meisten constant ist das Verhältniss zwischen $\mathbf{P}$ und $\mathbf{C}$, nämlich $10 \mathrm{C}: 1 \mathrm{P}$; nur bei der Guanylsäure ist das Verhältniss $11 \mathrm{C}: 1 \mathrm{P}$.

Die Erforschung der Guanylsäure in chemischer Beziehung ist zu Ende geführt. Wie man das Nucleoproteid Hammar sten's aus Pancreas als das beststudirte Nucleoproteid ansehen muss, so hoffe ich durch meine Untersuchung der Guanylsäure, des Spaltungsproduktes dieses Nucleoproteids, die klassische Arbeit Hammarsten's in einer Beziehung zum Abschluss geführt zu haben. 